$64^{\text {ème }}$ Congrès de la SFCO, 03001 (2016)

DOI:10.1051/sfco/20166403001

(C) Owned by the authors, published by EDP Sciences, 2016

\title{
POSTER
}

\section{À propos d'un carcinome adénoïde kystique palatin}

\section{Bouchet $\mathrm{J}^{1}$, Marès $\mathbf{S}^{2}$, Bertolus $\mathbf{C}^{2}$, Hervé $\mathbf{G}^{3}$, Lescaille $\mathbf{G}^{1}$, Rochefort $\mathrm{J}^{1}$}

1 - Service d'Odontologie du Pr Descroix, Groupe Hospitalier Pitié Salpêtrière-Charles Foix, UFR Odontologie Université Paris Diderot

2 - Service de Stomatologie et Chirurgie Maxillo-Faciale du Pr Goudot, Groupe Hospitalier Pitié Salpêtrière-Charles Foix, Université Pierre et Marie Curie

3 - Service d'Anatomie et de Cytologie pathologique du Pr Capron, Groupe Hospitalier Pitié Salpêtrière-Charles Foix, Université Pierre et Marie Curie

\section{Introduction}

Les carcinomes adénoïdes kystiques, anciennement appelées cylindromes, sont des tumeurs épithéliales malignes à croissance généralement lente, se développant le plus souvent aux dépens des glandes salivaires accessoires (1). Cette pathologie concerne surtout les patients de 40 à 60 ans, sans prépondérance de sexe. II n'existe pas à ce jour de facteur de risque connu. II s'agit du deuxième type de tumeurs les plus couramment retrouvées au niveau des glandes salivaires. Elles peuvent atteindre l'ensemble des glandes salivaires mais $50 \%$ à $60 \%$ se développent au niveau des glandes salivaires accessoires dont le site de prédilection est le palais (2).

\section{Observation}

Une femme de 77 ans, atteinte d'hypercholestérolémie, d'hypertension et d'une thrombocytémie essentielle (avec crise d'angor en 2010) traitée au long cours par Hydrea ${ }^{\circledR}$, Hyperium $^{\circledR}$, Kardegic $^{\circledR}$ et vitamine $\mathrm{D}$, a consulté initialement dans le service d'odontologie du groupe hospitalier La Pitié Salpêtrière pour une réhabilitation implantaire. Cette patiente présentait une intoxication tabagique évaluée à 40 paquet année. L'examen clinique révélait une tuméfaction palatine souple, inhomogène, intéressant le palais dur gauche, de $1 \mathrm{~cm}$ de grand axe, évoluant de manière asymptomatique depuis 20 ans sans adénopathie cervicale associée. Une biopsie a été réalisée. L'examen anatomopathologique a retrouvé une lésion cribriforme et tubuleuse évoquant un carcinome adénoïde kystique. La patiente a été adressée en chirurgie maxillo-faciale pour prise en charge carcinomateuse. Un bilan d'extension a été réalisé. L'IRM montrait une lésion grossièrement ovalaire du palais paramédian gauche, respectant la corticale sans rehaussement suspect des foramina basaux ou des nerfs intracrâniens, hypermétabolique intense au TEP. Àce jour, la patiente est en attente d'une exérèse chirurgicale sans évidement cervical.

\section{Discussion}

La présentation clinique est classiquement liée aux modalités d'extension particulière aux carcinomes adénoïdes kystiques : les cellules tumorales ont la capacité d'infiltrer la gaine des nerfs adjacents à la tumeur et de s'y propager. Cependant ceux-ci sont le plus souvent frustres, ce qui explique le fréquent retard au diagnostic. Les stades T3 et T4 initiaux représentent plus 
de $75 \%$ des cas, et si les métastases ganglionnaires et à distance sont rares au moment du diagnostic, il existe un risque ultérieur non négligeable de récidive locale et d'essaimage à distance : $40 \%$ des patients présenteront ainsi des métastases au cours de l'évolution de la maladie (3/4).

\section{Conclusion}

Devant ce type de lésion, une biopsie est indispensable et doit être réalisée dans les plus brefs délais. Le chirurgien oral doit connaitre les éléments diagnostiques caractéristiques ainsi que les modalités de prise en charge adaptées, permettant l'orientation rapide des patients auprès des services spécialisés.

\section{Références}

1. Barnes L, Eveson JW, Reichart P, Sidransky D, et al. Salivary glands. World Health Organization classification of tumours. Pathology and genetics of head and neck tumours. 2005:209-281.

2. Bonfils P. Tumeurs des glandes salivaires. EMC (Elsevier Masson SAS, Paris), Oto-rhino-laryngologie, 20-628-B10,2007.

3. Rhee CS, Won TB, Lee CH, Min YG, Sung MW, Kim KH, Shim WS, Kim YM, Kim JW. Adenoid cystic carcinoma of the sinonasal tract : treatment results. Laryngoscope. 2006 Jun;116(6):982-6.

4. Lupinetti AD, Roberts DB, Williams MD, Kupferman ME, Rosenthal DI, Demonte F, El-Naggar A, Weber RS, Hanna EY. Sinonasal adenoid cystic carcinoma: the M. D. Anderson Cancer Center experience. Cancer. 2007 Dec 15;110(12):272631. 